\title{
A New High-Performance Gadonanotube-Polymer Hybrid Material for Stem Cell Labeling and Tracking by MRI
}

\author{
Sakineh E. Moghaddam, ${ }^{1,2}$ Mayra Hernández-Rivera $\mathbb{D D}^{1,2}$ Nicholas G. Zaibaq $\mathbb{D}{ }^{1}$ \\ Afis Ajala $\mathbb{D}^{3,4}$ Maria da Graça Cabreira-Hansen, ${ }^{2}$ Saghar Mowlazadeh-Haghighi $\mathbb{D}^{1,2}$ \\ James T. Willerson, ${ }^{2}$ Emerson C. Perin, ${ }^{2}$ Raja Muthupillai $\mathbb{D}^{3},{ }^{3}$ and Lon J. Wilson $\mathbb{D}^{1}$ \\ ${ }^{1}$ Department of Chemistry, The Smalley-Curl Institute, Rice University, M.S. 60, P.O. Box 1892, Houston, \\ TX 77251-1892, USA \\ ${ }^{2}$ Stem Cell Center, Texas Heart Institute, Baylor St. Luke's Medical Center, MC 2-255, P.O. Box 20345, Houston, \\ TX 77225-0345, USA \\ ${ }^{3}$ Department of Radiology, Baylor St. Luke's Medical Center, 6720 Bertner Avenue, MC 2-270, Houston, \\ TX 77030-2697, USA \\ ${ }^{4}$ Department of Physics, University of Houston, Houston, TX 77004, USA
}

Correspondence should be addressed to Lon J. Wilson; durango@rice.edu

Received 6 February 2018; Accepted 19 April 2018; Published 10 July 2018

Academic Editor: Gaurav Malviya

Copyright ( 2018 Sakineh E. Moghaddam et al. This is an open access article distributed under the Creative Commons Attribution License, which permits unrestricted use, distribution, and reproduction in any medium, provided the original work is properly cited.

A gentle, rapid method has been developed to introduce a polyacrylic acid (PAA) polymer coating on the surface of gadonanotubes (GNTs) which significantly increases their dispersibility in water without the need of a surfactant. As a result, the polymer, with its many carboxylic acid groups, coats the surface of the GNTs to form a new GNT-polymer hybrid material (PAAGNT) which can be highly dispersed in water (ca. $20 \mathrm{mg} \cdot \mathrm{mL}^{-1}$ ) at physiological $\mathrm{pH}$. When dispersed in water, the new PAA-GNT material is a powerful MRI contrast agent with an extremely short water proton spin-lattice relaxation time $\left(T_{1}\right)$ which results in a $T_{1}$-weighted relaxivity of $150 \mathrm{mM}^{-1} \cdot \mathrm{s}^{-1}$ per $\mathrm{Gd}^{3+}$ ion at $1.5 \mathrm{~T}$. Furthermore, the PAA-GNTs have been used to safely label porcine bone-marrow-derived mesenchymal stem cells for magnetic resonance imaging. The labeled cells display excellent image contrast in phantom imaging experiments, and transmission electron microscopy images of the labeled cells reveal the presence of highly dispersed PAA-GNTs within the cytoplasm with $10^{14} \mathrm{Gd}^{3+}$ ions per cell.

\section{Introduction}

Among the many nanoparticles currently under investigation, carbon nanotubes (CNTs) have been and continue to be a key material because of their unique properties and extreme diversity of potential applications [1-5]. However, poor solubility of CNTs in water or organic solvents is a challenging limitation for many practical applications of this unique material [6]. To integrate CNT technology with medical and biological milieu, CNT solubility or suspendability needs to be improved for aqueous and biological media [7-9]. Toward this end, the two main approaches that have been used to increase dispersion of CNTs are (1) noncovalent functionalization of CNTs with surfactants, nucleic acids, peptides, polymers, or oligomers [10-14] and (2) CNT covalent functionalization [15-17].

Nanomaterials, including CNTs, have been extensively studied as diagnostic agents, for example, as contrast agents (CAs) for magnetic resonance imaging (MRI). MRI has become a standard clinical tool to obtain human anatomical and functional information noninvasively [18-20]. In current clinical approaches, CAs are sometimes administered to enhance signal intensity of MR images [21-23]. Because $\mathrm{Gd}^{3+}$ has seven unpaired electrons, giving it a high magnetic moment and relatively long magnetic relaxation time, most of these $\mathrm{CA}$ materials are small-molecule $\mathrm{Gd}^{3+}$-chelate compounds that disseminate uniformly throughout the vasculature $[21,22,24,25]$. The efficacy of these MRI CAs is 
characterized by their relaxivity $\left(r_{1}\right)$, which is the water proton relaxation rate constant normalized to the concentration of the CA. These image-brightening agents shorten the water proton spin-lattice relaxation time $\left(T_{1}\right)$ by the magnetic coupling of their paramagnetic centers to the surrounding water proton nuclear spin [21, 22, 24]. Although most current clinically used $\mathrm{Gd}^{3+}$ ion-based CAs have acceptable safety profiles, they also possess very low relaxivities of only $\sim 4-5$ at $1.5 \mathrm{~T}$ and $37^{\circ} \mathrm{C}[26-28]$.

In 2005, gadonanotubes (GNTs) were first reported, revealing a new strategy for sequestering $\mathrm{Gd}^{3+}$ ions by encapsulating them within (or upon) ultrashort carbon nanotube capsules (US-tubes) [29]. This new carbon nanostructure with a relaxivity as high as $160 \mathrm{mM}^{-1} \cdot \mathrm{s}^{-1}$ per $\mathrm{Gd}^{3+}$ ion at $1.5 \mathrm{~T}$ and $37^{\circ} \mathrm{C}$ is the highest-performing $\mathrm{Gd}^{3+}$-based MRI CA material discovered to date [30]. US-tubes are produced by first cutting full-length single-walled carbon nanotubes (SWCNTs, $>1 \mu \mathrm{m}$ in length) via a previously reported fluorination/pyrolysis method [31]. Thereafter, US-tubes (average length of $50 \mathrm{~nm}$ ) are purified by treatment with $\mathrm{HCl}$, debundled under Birch reduction conditions, and then mildly oxidized by $\mathrm{HNO}_{3}$ to produce carboxylic acid groups at US-tube defect sites. Mild sonication of US-tubes in the presence of $\mathrm{Gd}^{3+}$ ions in aqueous solution is then the final step in the preparation of the GNTs. To increase the dispersibility of the GNTs in biological media for in vivo studies, they have often been suspended in aqueous solution using Pluronic ${ }^{\circledR}-108$ [32, 33], a nonionic and biocompatible surfactant. In further efforts to boost the dispersibility of GNTs in biological media, we recently produced a second-generation GNT material (PCP-GNTs) by covalently attaching benzoic acid groups via diazonium-based chemistry [34].

In the current study, we have produced yet a thirdgeneration GNT material by coating the GNT outer surface using a mild in situ polyacrylic acid (PAA) polymerization procedure to produce a new highly water-dispersible PAAGNT material. The motivation for producing this new material was twofold. First, surfactant-wrapped GNT materials are known to quickly shed the surfactant wrapping in vivo $[2,35-37]$, and it was hoped that the new PAA-GNT material would resist this process through stronger intermolecular attraction gained from using the GNTs as a scaffold for polymerization. By making GNTs water-soluble and stable in biological media, the potential to move this material toward vascular MRI applications for the first time is significantly greater. Secondly, the second-generation GNT material, with covalently attached benzoic acid groups (PCP-GNTs), is labor-intensive and time-consuming [34], and it was hoped that the new PAA-GNT material could be produced more quickly and using a much simpler synthetic process which has now been verified by the current study. Finally, we have also evaluated MRI performance of the new PAA-GNT material and employed the material to safely label and image porcine bone-marrow-derived mesenchymal stem cells (MSCs) as a demonstration of a valuable application for the material.

\section{Experimental}

2.1. Preparation of the PAA-GNTs. We followed the methods of Gizzatov et al., and US-tubes were prepared by a previously reported method $[31,34]$. Briefly, $200 \mathrm{mg}$ of SWCNTs (Carbon-Arc SWCNTs from Carbon Solutions Inc.) was fluorinated using $2 \% \mathrm{~F}_{2}$ in a $\mathrm{He}$ gas mixture with a flow rate adjusted to $15 \mathrm{~cm}^{3} \cdot \mathrm{min}^{-1}$ along with $\mathrm{H}_{2}$ gas at a flow rate of $10 \mathrm{~cm}^{3} \cdot \mathrm{min}^{-1}$ at $125^{\circ} \mathrm{C}$ for $2.5 \mathrm{~h}$. The fluorinated product was then heated at $1000^{\circ} \mathrm{C}$ for $3 \mathrm{~h}$ under a continuous flow of Ar. The as-produced US-tubes were then sonicated in $200 \mathrm{~mL}$ of concentrated $\mathrm{HCl}$ for 60 min to remove metal impurities, washed with $\mathrm{DI} \mathrm{H}_{2} \mathrm{O}$, dried, and individualized by sonication for $60 \mathrm{~min}$ in $200 \mathrm{~mL}$ of dry THF and $\mathrm{Na}^{0}$ of equal weight to the US-tube sample. Next, US-tubes were refluxed in $200 \mathrm{~mL}$ of $6 \mathrm{M} \mathrm{HNO}_{3}$ for $15 \mathrm{~min}$, washed with $\mathrm{DI} \mathrm{H}_{2} \mathrm{O}$, and dried. Loading of the US-tubes with $\mathrm{GdCl}_{3}$ was achieved by $1 \mathrm{~h}$ sonication in a $1 \mathrm{mM}$ aqueous solution of $\mathrm{GdCl}_{3}$ to produce Gd@US-tubes or gadonanotubes (GNTs). The GNT product was washed with $\mathrm{DI} \mathrm{H}_{2} \mathrm{O}$ until $\mathrm{Gd}^{3+}$ ions could not be detected in the filtrate (as determined by inductively coupled plasma optical emission spectrometry, or ICP-OES). GNTs were then further functionalized using an in situ polymer growth procedure. Briefly, $100 \mathrm{mg}$ GNTs in $40 \mathrm{~mL} \mathrm{H}_{2} \mathrm{O}$ was added to a $100 \mathrm{~mL}$ 3-neck flask. The mixture was sonicated for $30 \mathrm{~min}$ at $12 \mathrm{~W}$ and $55 \mathrm{kHz}$, and the well-suspended mixture was stirred vigorously at $50^{\circ} \mathrm{C}$ under $\mathrm{N}_{2}$ gas. Next, a solution of $1.5 \mathrm{mg}$ potassium persulfate (KSP) dissolved in $200 \mathrm{mg}$ acrylic acid was added with a tube pump at $5 \mathrm{~mL} \cdot \mathrm{h}^{-1}$, and the mixture was then let stir for $3 \mathrm{~h}$. After completion of the reaction, the suspension was filtered through a $0.2 \mu \mathrm{m}$ PTFE membrane and the collected powder was redispersed in water with stirring for one day before being filtered. Finally, the resultant powder was dried in a vacuum oven at $80^{\circ} \mathrm{C}$ overnight to obtain the PAA-GNT sample. The maximum suspendability of the PAA-GNTs in water was determined to be ca. $20 \mathrm{mg} \cdot \mathrm{mL}^{-1}$ by lyophilizing an aliquot of a supersaturated solution in which an excess of the PAA-GNTs was dispersed in $2 \mathrm{~mL}$ of $\mathrm{H}_{2} \mathrm{O}$ and left undisturbed for $24 \mathrm{~h}$, after which $400 \mu \mathrm{L}$ of the supernatant solution was dried and the dry product (powder) was weighted using a microbalance. The obtained PAA-GNT material was then characterized with high-resolution transmission electron microscopy (HRTEM, JEOL 2100), scanning electron microscope equipped with energy dispersive spectrometry (EDS, FEI Quanta 400F), ICPOES using a Perkin-Elmer Optima 8300 instrument, Raman spectroscopy using a ReNishaw inVia Raman microscope, and thermogravimetric analysis (TGA) using a Q-600 simultaneous TGA/DSC from TA Instruments. In vitro work was performed using PAA-GNTs which contained ca. $4.5 \%$ Gd by weight as determined by ICP-OES.

2.2. MR Imaging and Relaxometric Analysis. Phantom MR images of the PAA-GNT CAs were prepared by taking a $0.9 \mathrm{mg} \mathrm{mL}^{-1}$ aqueous dispersion of each sample. $T_{1^{-}}$ weighted MR images of the samples were then determined at room temperature $(\mathrm{RT})\left(25^{\circ} \mathrm{C}\right)$ using a commercial $1.5 \mathrm{~T}$ MRI scanner (Achieva, Philips Medical System, the Netherlands). A Q-body coil and an 8-channel wrist coil were used for radio-frequency transmission and signal reception, respectively. An inversion recovery prepared turbospin sequence was used to measure the $T_{1}$ relaxation times of the 
samples $(\mathrm{TR}=10000 \mathrm{~ms} ; \mathrm{TE}=8 \mathrm{~ms})$. The images were acquired over a field of view of $81 \times 121 \mathrm{~mm}$, with an acquired voxel resolution of $0.59 \times 0.77 \times 5.00 \mathrm{~mm}$ and a reconstruction matrix resolution of $0.24 \times 0.24 \times 5.00 \mathrm{~mm}$. Following the inversion preparation, data acquisition commenced after inversion delay times (TI) of 200, 400, 800, 1200 , and $1500 \mathrm{~ms}$, and the $T_{1}$ values were calculated using the standard inversion recovery equation. HPLC-grade water was used as a diamagnetic control. The samples were then digested in $26 \% \mathrm{HClO}_{3}$ and reconstituted in $10 \mathrm{~mL}$ of trace metal-grade $2 \% \mathrm{HNO}_{3}(\mathrm{aq})$ for determination of $\mathrm{Gd}^{3+}$ ion concentration by ICP-OES.

2.3. Stem Cell Labeling Experiments. The PAA-GNTs were used to intracellularly label porcine bone-marrow-derived mesenchymal stem cells (MSCs) harvested from three different animals. To prepare the stock labeling solution, the PAAGNTs were suspended in water $\left(100 \mu \mathrm{M} \mathrm{Gd^{3+ }}\right.$, by ICP-OES) and the suspension was sterilized by UV light exposure for $3 \mathrm{~h}$ with rocking, which has been shown to be a procedure that does not cause damage to CNT materials [38]. MSCs were grown in T-175 flasks with alpha minimal essential medium ( $\alpha \mathrm{MEM})$ containing $10 \%$ fetal bovine serum (FBS) and incubated at $37^{\circ} \mathrm{C}$ (95\% relative humidity in $5 \% \mathrm{CO}_{2}$ in air). Cells were expanded until the third passage prior to labeling. The PAA-GNT-labeled MSCs were prepared by adding the stock labeling solution

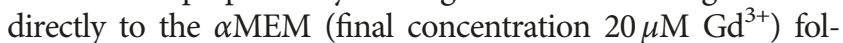
lowed by incubation of the cells for $24 \mathrm{~h}$ with the CA. After collecting the cells, the suspension was passed through a $70 \mu \mathrm{m}$ nylon filter to eliminate cell aggregates and the cells were resuspended in $20 \mathrm{~mL}$ of $\alpha \mathrm{MEM}$. A known density gradient separation method was applied using a $50 \mathrm{~mL}$ conical tube to isolate the cells from excess of PAA-GNTs in solution, as well as from "heavy" cells which are labeled cells with PAA-GNTs on their cellular membrane [2]. To accomplish this, $10 \mathrm{~mL}$ of FicollPaque $\left(20^{\circ} \mathrm{C}\right.$, Sigma-Aldrich) was added to the bottom of the conical tube containing $20 \mathrm{~mL}$ of the cell suspension and the sample was centrifuged at $400 \mathrm{~g}$ for $20 \mathrm{~min}$. The labeled MSCs were then isolated from the interface of the $\alpha \mathrm{MEM}$ and the Ficoll-Paque using a plastic transfer pipette. Cells were then resuspended in fresh $\alpha \mathrm{MEM}$ and centrifuged at $1500 \mathrm{rpm}$ for 10 min to wash the residue of Ficoll-Paque in cells. Cell counts were obtained using a Beckman Counter MultiSizer 3. Unlabeled MSCs were used as control cells. Aliquots of labeled and unlabeled cell suspensions were collected and analyzed by ICPOES (Perkin-Elmer Optima 8300 instrumentation) to determine the $\mathrm{Gd}^{3+}$ ion concentration in the cells. To prepare the samples, cells were heated and treated with two alternating additions of $500 \mu \mathrm{L} 70 \%$ trace metal-grade $\mathrm{HNO}_{3}$ and $26 \%$ $\mathrm{HClO}_{3}$, allowing the samples to dry between additions. Finally, the samples were diluted to $10 \mathrm{~mL}$ with an aqueous solution of $2 \%$ trace metal-grade $\mathrm{HNO}_{3}$ and $2 \%$ ethanol and finally filtered through a $0.22 \mu \mathrm{m}$ pore size syringe filter.

2.4. Viability of the PAA-GNT-Labeled MSCs. Fluorescenceactivated cell sorting (FACS) was performed using a BD Biosciences LSRII Analyzer in order to determine the viability of the MSCs after being exposed to PAA-GNTs for $24 \mathrm{~h}$. The experiment was run in triplicate using three different animal cell lines. A LIVE/DEAD viability/cytotoxicity assay kit (Life Technologies) was used to stain the cells: green-fluorescent calcein-AM indicates intracellular esterase activity in viable cells, while red-fluorescent ethidium homodimer-1 indicates dead cells when the cell membrane was compromised. Unlabeled MSCs were used as the positive control while unlabeled MSCs incubated with $70 \%$ methanol for 15 min were used as the negative control (dead cells). The dyes were added, and the samples were incubated in the dark at room temperature for $20 \mathrm{~min}$ prior to analysis.

2.5. MR Imaging of the PAA-GNT-Labeled MSCs. PAA-GNT-labeled MSCs were prepared as described above. Samples of 30 million unlabeled and labeled MSCs were separately centrifuged in a $1.5 \mathrm{~mL}$ Eppendorf tube to form cell pellets. The supernatant was carefully removed without disturbing the cell pellet. Cautiously, $500 \mu \mathrm{L}$ of $0.5 \%$ agar was added on top of cell pellet. The $T_{1}$-weighted MR images of the labeled and unlabeled MSCs were acquired at RT using a commercial 1.5 T MRI scanner (Achieva, Philips Medical System, the Netherlands) with an inversion recovery prepared spin echo sequence (acquisition voxel size: $1.1 \times 1.1 \times 5 \mathrm{~mm}^{3}$; TR/TE: $6000 \mathrm{~ms} / 11 \mathrm{~ms})$. The experiment was repeated at various inversion times (TIs): 50, 100, 200, 400, 600, 800, 1200, 2000, 3000, and $4000 \mathrm{~ms}$.

2.6. Transmission Electron Microscopy (TEM) Imaging of the PAA-GNT-Labeled MSCs. TEM analysis was performed to determine the subcellular localization of the PAA-GNT CAs. Labeled MSCs and unlabeled MSCs were centrifuged separately at $1500 \mathrm{rpm}$ for $10 \mathrm{~min}$ to form a cell pellet. Without disturbing the pellet, the supernatant was removed, $3 \%$ glutaraldehyde was added, and the samples were left undisturbed for 2 days. Later, the samples were washed with $1 \mathrm{X}$ phosphate-buffered saline (PBS) and postfixed with $1 \%$ $\mathrm{OsO}_{4}$ for $1 \mathrm{~h}$ and then washed and dehydrated with increasing concentration of ethanol, and infiltrated with acetone and Epon 812 resin. Finally, the samples were embedded with $100 \%$ Epon 812 in a mold, cut into $1 \mathrm{~mm}$ sections, and stained with $1 \%$ methylene blue and $1 \%$ basic fuchsin. Ultrathin sections of $80 \mathrm{~nm}$ were cut from the sample block using a Leica EM UC7 ultramicrotome and framed on 100-mesh copper grids. Grids were stained with $2 \%$ alcoholic uranyl acetate and Reynold's lead citrate. The grids were examined using a JEOL 1230 TEM instrument equipped with an AMTV 600 digital imaging system at the Texas Heart Institute (Houston, TX).

\section{Results and Discussion}

3.1. Characterization of PAA-GNTs. GNTs were prepared as previously reported [29]. The concentration of the $\mathrm{Gd}^{3+}$ ions of the GNTs was determined to be 4.5 wt.\% by ICP-OES. The surface of the GNTs was then functionalized via an in situ free radical polymerization of acrylic acid (AA), using potassium persulfate (KSP) as an initiator to prepare the 


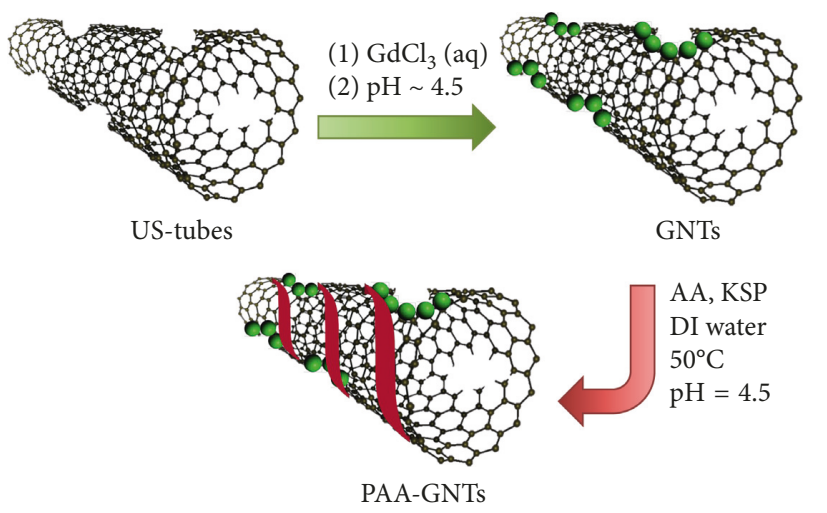

FIGURE 1: Schematic representation for the preparation of the PAA-GNTs.

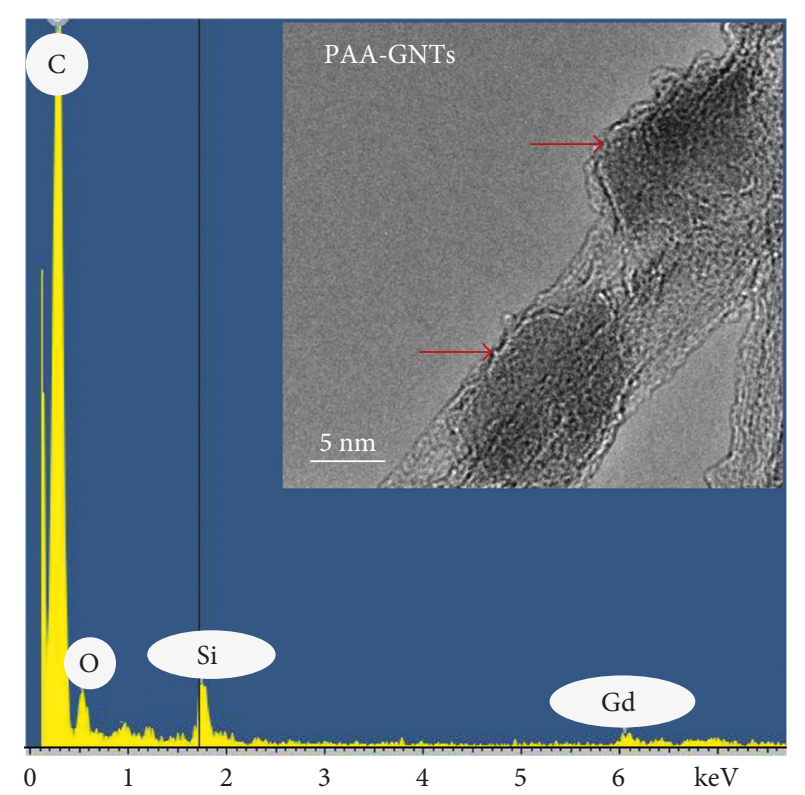

FIGURE 2: EDS data for the PAA-GNTs; inset: HRTEM image of the PAA-GNTs.

resultant PAA-GNTs (Figure 1). To obtain the greatest dispersibility for the PAA-GNTs, control reactions were performed using empty US-tubes under different reaction conditions.

Dispersibility testing revealed that, by increasing the concentration of AA monomer to $0.17 \mathrm{M}$, the dispersibility of the PAA-GNT product was increased up to 40 times compared to untreated US-tubes. However, a further increase in the concentration of AA led to a higher degree of polymerization, which, in turn, reduced the suspendability of the PAA-GNT product in water. The highest degree of dispersibility for the PAA-GNTs was achieved by using a 5:1 weight ratio of AA to US-tubes. Furthermore, the $\mathrm{pH}$ of the reaction had to be maintained above $\mathrm{pH} 4.5$ to prevent loss of $\mathrm{Gd}^{3+}$ ions from the PAA-GNT product, which began to occur by $\mathrm{pH} 4.0$ [39]. Under these conditions, the PAA-GNT product contained 4.5 wt.\% Gd.
The TEM image of the PAA-GNT material (Figure 2, inset) showed that the general structure of the US-tubes was preserved after the in situ radical polymerization procedure to produce the PAA-GNTs. The darker areas of the inset of Figure 2, as indicated by the red arrows, suggest the presence of polymer on/around the GNTs. As expected, electron dispersive spectroscopy (EDS), also shown in Figure 2, demonstrated the presence of carbon, oxygen, and gadolinium for the PAA-GNT product. The silicon peak is an artifact related to the Si content of the EDS detector [40].

TGA data for the PAA-GNTs were used to confirm the presence of US-tubes wrapped with PAA polymer, as shown in Figure 3(a). TGA profiles showed greater weight loss with increasing temperature for samples with PAA content (PAAUS-tubes and PAA-GNTs) compared to the US-tubes alone. The first weight loss took place mostly in the $200-500^{\circ} \mathrm{C}$ range, probably because of decarboxylation either from the carboxylate groups at US-tube or GNT defect sites or from the PAA coating. Comparative TGA profiles for the US-tubes, PAA-US-tubes, and PAA-GNTs provided valuable information about the presence of PAA in the modified structures. Greater weight loss in the temperature range of $200-500^{\circ} \mathrm{C}$ for the PAA-US-tubes (17 wt.\%) and PAA-GNTs (15 wt.\%) versus only a $12 \mathrm{wt} . \%$ loss for US-tubes indicates the presence of additional carboxylate groups from the PAA coating for those structures. Raman spectra of the PAA-UStubes and PAA-GNTs, shown in Figure 3(b), have the characteristic $D, G, G^{\prime}$, and RBM bands of CNT materials, with the PAA-containing samples showing somewhat greater intensity for all the bands compared to the US-tubes alone.

3.2. Relaxivity and MRI Performance of PAA-GNTs. To establish their properties as MRI CAs, aqueous dispersion of PAA-GNTs and PAA-US-tubes at a concentration of $0.9 \mathrm{mg} \cdot \mathrm{mL}^{-1}$ was imaged using a $1.5 \mathrm{~T}$ MRI scanner. Due to poor dispersibility of US-tube and GNT samples, they were suspended in a $0.17 \mathrm{v} / \mathrm{w} \%$ aqueous solution of Pluronic-108 surfactant for imaging. $T_{1}$-weighted MR phantom images acquired using a $600 \mathrm{~s}$ inversion time (TI) demonstrated that there is clear visual contrast difference between controls with no $\mathrm{Gd}^{3+}$ (US-tubes and PAA-US-tubes) and the GNTs and PAA-GNTs, as shown in Figure 4.

Relaxivities for the functionalized PAA-GNTs and controls were calculated from the evolution of MR signal acquired at different inversion delays. The $r_{1}$ value for the PAA-GNTs was $150 \mathrm{mM}^{-1} \cdot \mathrm{s}^{-1}$ which is comparable to the relaxivity value of GNTs reported previously [29]. This relaxivity for the PAA-GNTs in water suggests high dispersibility of the material, eliminating the need for a surfactant to achieve highly suspendable GNTs. Studies to determine the stability of $\mathrm{Gd}^{3+}$ within the PAA-GNTs were also performed which demonstrated that a challenge with $10 \%$ fetal bovine serum (FBS) in phosphate-buffered solution (PBS) did not produce any loss of $\mathrm{Gd}^{3+}$ ion after a $24 / 48 \mathrm{~h}$ challenge period (Figure $1 \mathrm{~S}$ ).

3.3. Cell Viability and MRI Studies of the PAA-GNT-Labeled MSCs. Before evaluating the performance of the PAA-GNT 


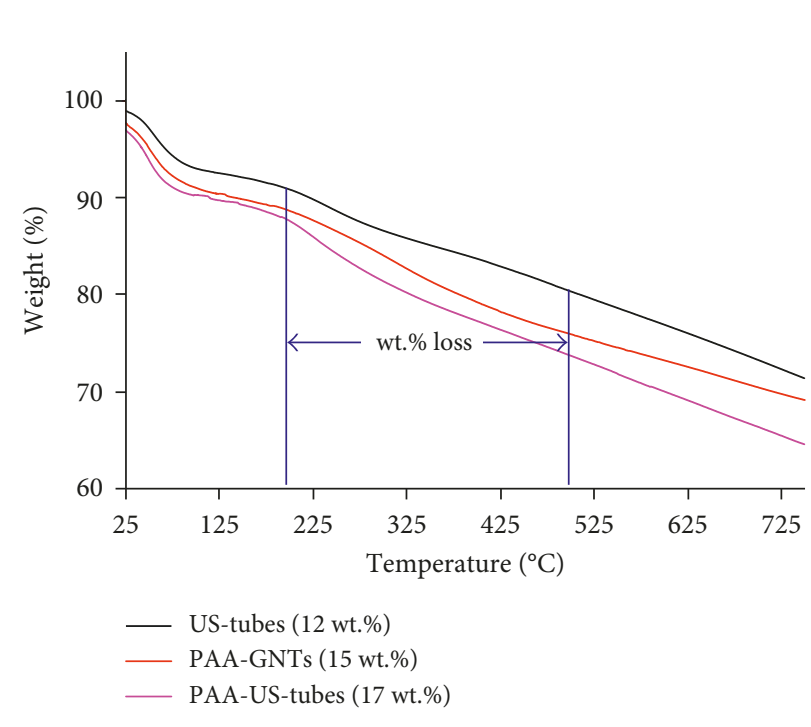

(a)

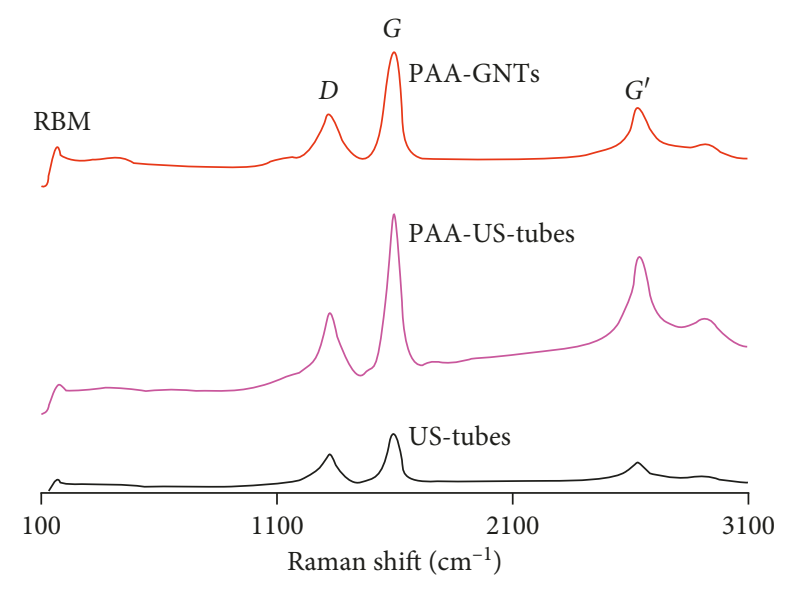

(b)

FIgUre 3: (a) TGA data and (b) Raman spectroscopy data for the US-tubes, PAA-US-tubes, and PAA-GNTs.

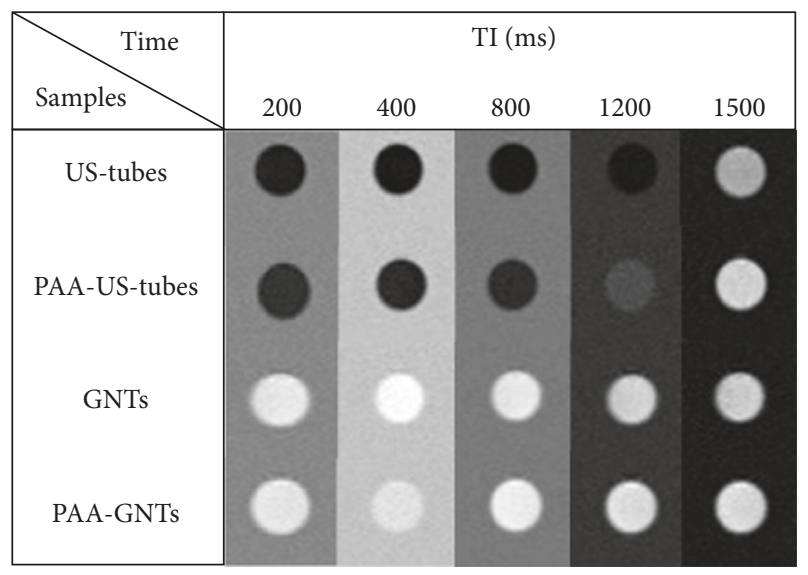

Figure 4: $T_{1}$-weighted MR phantom images of aqueous dispersion of samples $(0.9 \mathrm{mg} / \mathrm{mL})$ acquired at $1.5 \mathrm{~T}$ and $\mathrm{RT}$ with different inversion times $(\mathrm{TI})$.

material as an intracellular CA (Figure 5), its cytotoxicity in MSCs was examined using FACS analysis to determine the viability of labeled cells compared to unlabeled control cells. After incubating MSCs with PAA-GNTs $\left(20 \mu \mathrm{M} \mathrm{Gd}^{3+}\right)$ for $24 \mathrm{~h}$, the uptake of PAA-GNTs by the cells was confirmed and quantified by ICP-OES analysis. Approximately $10^{14}$ $\mathrm{Gd}^{3+}$ ions/cell were successfully taken up, which is a significantly higher concentration of $\mathrm{Gd}^{3+}$ ions/cell than was previously taken up using Pluronic-wrapped GNTs $\left(10^{9} \mathrm{Gd}^{3+}\right.$ ions/cell) [2]. Cytotoxicity studies using FACS (Figure 2S) showed no difference in viability measured by calcein staining, demonstrating that the membrane integrity of the cells was not compromised and that the MSCs remained highly viable after 24 hours of exposure to PAA-GNTs. As shown in Supplemental Figure 3S, the percentage of dead cells was $2.8 \%(\mathrm{SEM}=0.7)$ in control samples; meanwhile, the PAA-GNT samples showed 2.1\% $(\mathrm{SEM}=0.3)$. After

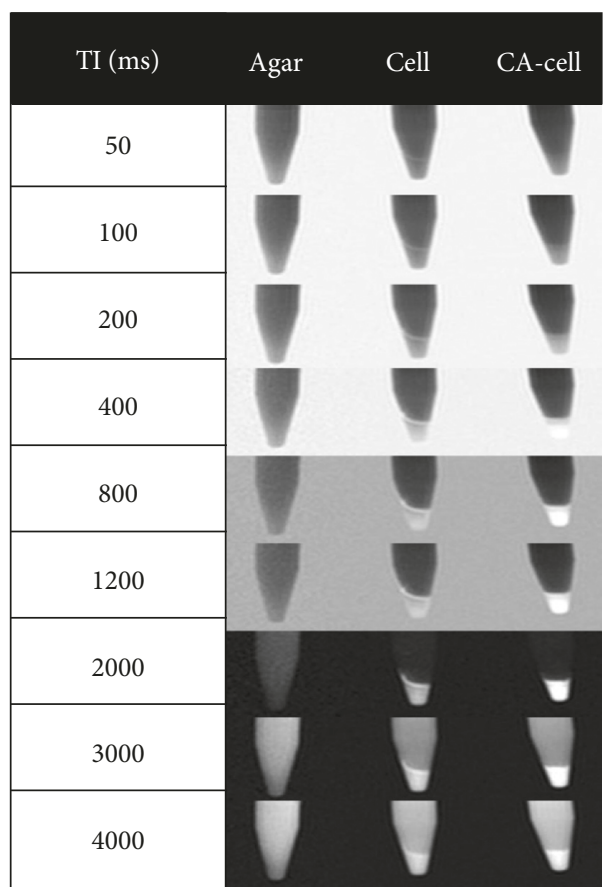

FIgURE 5: $T_{1}$-weighted MR images of the samples acquired at $1.5 \mathrm{~T}$ and RT. Left to right: agar in water (agar), control sample of $3 \times 10^{7}$ unlabeled MSCs (cell), and $3 \times 10^{7}$ PAA-GNTs-labeled MSCs (CAcell). All cell-containing samples were in a $0.5 \%$ agarose gel.

demonstrating that PAA-GNTs can be internalized into MSCs to deliver a high concentration of $\mathrm{Gd}^{3+}$ ions/cell safely, MR images of the labeled MSCs were obtained. Agarose gel $(0.5 \mathrm{~mL}$ of $0.5 \%)$ was added on the top of a pellet containing $3 \times 10^{7}$ PAA-GNT-labeled and unlabeled MSCs. The resulting MSC pellets and a water phantom were then imaged using a $1.5 \mathrm{~T}$ MRI scanner. The $T_{1}$-weighted MR images clearly demonstrate the rapid MR signal recovery of labeled cells compared to unlabeled cells and water phantom 

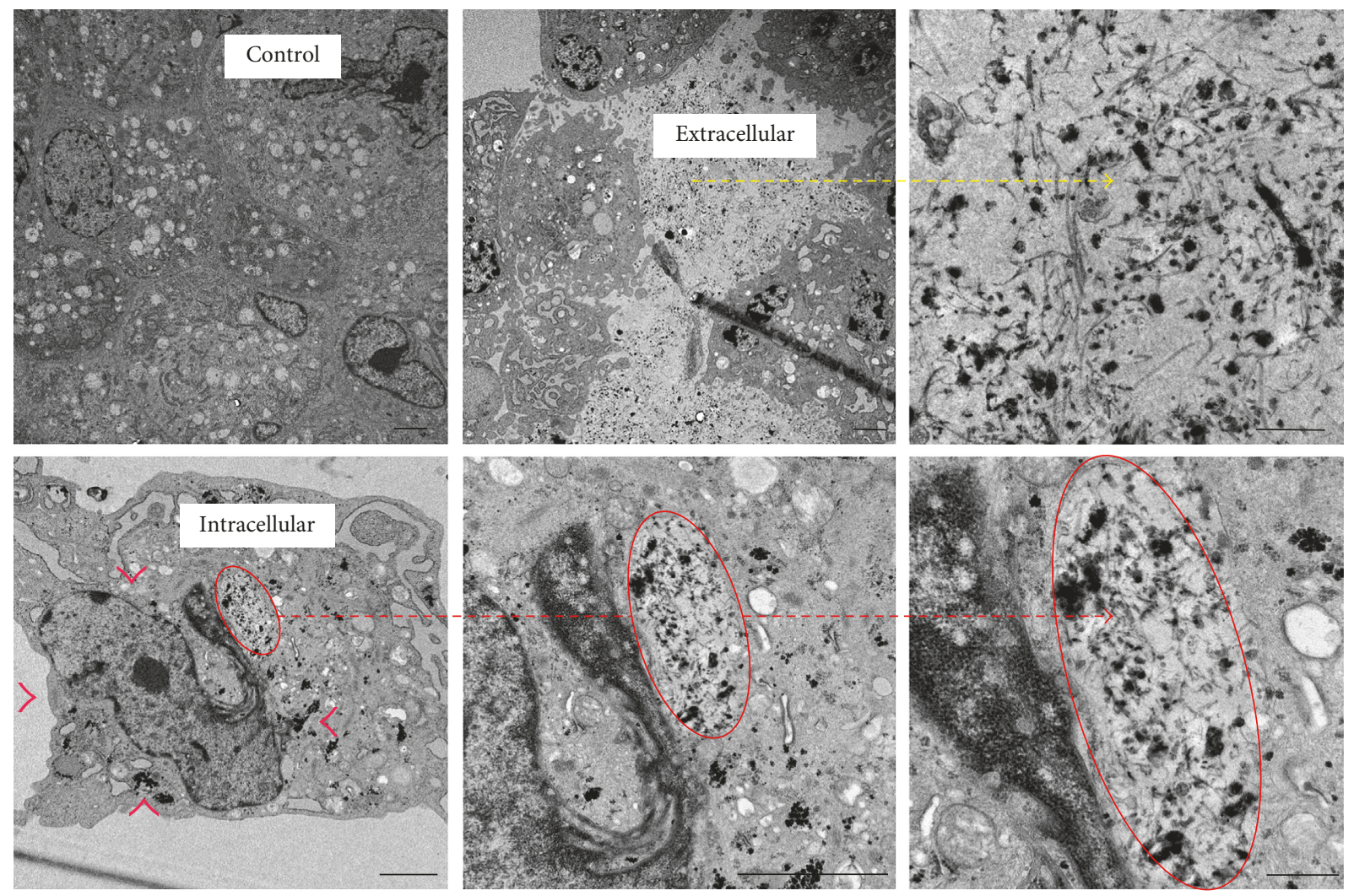

FIgURE 6: Representative TEM images of MSCs labeled with PAA-GNTs. Red circles indicate the intracellular PAA-GNTs localized in the cytoplasm of the cells, red arrowheads show scattered PAA-GNTs, and the yellow arrow shows PAA-GNTs in the extracellular space. Scale bars $=2 \mu \mathrm{m}$.

images (Figure 5), which is especially conspicuous in images acquired at TIs in the range of $800-3000 \mathrm{~ms}$.

TEM images of the PAA-GNT-labeled MSCs demonstrated that the PAA-GNTs appear as an accumulation of mostly small separated bundles of electron-dense aggregates of PAA-GNTs within the MSCs (Figure 6). In general, the material is not encapsulated within vesicles but appears to accumulate and aggregate in small clusters/bundles within the cytoplasm. From the TEM images, it is also apparent that PAA-GNTs do not enter the nucleus, which is preferable since foreign materials within the nucleus could interact and adversely alter DNA within cells.

The mostly very small bundles of PAA-GNTs found in the cytoplasm of MSCs in the present study are strikingly different from the very large bundles observed in the cytoplasm after labeling with surfactant-wrapped GNTs in our previous study [2]. We suggest that this notable difference may be due to the fact that the surfactant coating of surfactant-wrapped GNTs may be stripped off during the cell labeling process, which, in turn, encourages aggregation of the GNTs once they are internalized in the cell. Since the PAA coating of the PAA-GNTs appears to be stable in cells, it may be that there is some enhanced intermolecular interaction created between the coating and the GNTs that keeps it firmly attached when the AA polymerizes on the surface of the GNTs which might involve the GNT carboxylic acid groups at the defect sites. Thus, the PAA-GNTs appear to be a superior cell labeling agent compared to surfactantwrapped GNTs with better dispensability in biological media which results in greater cellular uptake with $10^{14} \mathrm{Gd}^{3+}$ ions/cell versus $10^{9}$ ion/cell for surfactant-wrapped GNTs [2].

\section{Conclusion}

In summary, this work has demonstrated that in situ surface polymerization of acrylic acid onto GNTs produces a highly water-dispersible counterpart, the PAA-GNTs, while maintaining the same relaxivity as surfactant-wrapped GNTs $\left(150 \mathrm{mM}^{-1} \cdot \mathrm{s}^{-1}\right)$. The PAA-GNT material can be dispersed in aqueous solution to the extent of approximately $20 \mathrm{mg} \cdot \mathrm{mL}^{-1}$ without the use of a surfactant. Furthermore, it has been shown that the PAA-GNT CAs can be safely used to internally label porcine bone-marrow-derived MSCs to visualize the cells with MRI with potential applications for monitoring transplanted stem cells in vivo. Due to the enhanced stability in aqueous solution as well as in cells without the need of a surfactant, this new, highly waterdispersible PAA-GNT material appears to be a better cell labeling agent than surfactant-wrapped GNTs.

\section{Data Availability}

The data used to support the findings of this study are available from the corresponding author upon request. 


\section{Conflicts of Interest}

There are no conflicts of interest to declare.

\section{Acknowledgments}

Sakineh E. Moghaddam, Mayra Hernández-Rivera, and Lon J. Wilson gratefully acknowledge the Welch Foundation (Grant C-0627 to Lon J. Wilson) and the National Science Foundation Graduate Research Fellowship Program (1450681 to Nicholas G. Zaibaq). The authors also thank Allan Prejusa for flow cytometry analysis and Ralph Nichols for acquiring the TEM images (Texas Heart Institute at Baylor St. Luke's Episcopal Hospital, Houston, TX, USA) and Dr. Maximillian Buja (University of Texas Health Science Center of Houston, Houston, TX, USA) for his assistance with TEM analysis.

\section{Supplementary Materials}

Supplementary information includes details on how the material was challenged for stability in biological media over time (Figure 1S), cell viability studies (Figure $2 S$ ), and average dead cell population frequency (Figure 3S) of control and material-treated MSCs. (Supplementary Materials)

\section{References}

[1] M. Hernández-Rivera, N. G. Zaibaq, and L. J. Wilson, "Toward carbon nanotube-based imaging agents for the clinic," Biomaterials, vol. 101, pp. 229-240, 2016.

[2] L. A. Tran, R. Krishnamurthy, R. Muthupillai et al., "Gadonanotubes as magnetic nanolabels for stem cell detection," Biomaterials, vol. 31, no. 36, pp. 9482-9491, 2010.

[3] S. Abdalla, F. Al-Marzouki, A. A. Al-Ghamdi, and A. AbdelDaiem, "Different technical applications of carbon nanotubes," Nanoscale Research Letters, vol. 10, no. 1, p. 358, 2015.

[4] C. Gao, Z. Guo, J.-H. Liu, and X.-J. Huang, "The new age of carbon nanotubes: an updated review of functionalized carbon nanotubes in electrochemical sensors," Nanoscale, vol. 4, no. 6, pp. 1948-1963, 2012.

[5] M. Hernández-Rivera, I. Kumar, S. Y. Cho et al., "Highperformance hybrid bismuth-carbon nanotube based contrast agent for X-ray CT imaging," ACS Applied Materials and Interfaces, vol. 9, no. 7, pp. 5709-5716, 2017.

[6] D. Tasis, N. Tagmatarchis, A. Bianco, and M. Prato, "Chemistry of carbon nanotubes," Chemical Reviews, vol. 106, no. 3, pp. 1105-1136, 2006.

[7] L. Lacerda, A. Bianco, M. Prato, and K. Kostarelos, "Carbon nanotubes as nanomedicines: from toxicology to pharmacology," Advanced Drug Delivery Reviews, vol. 58, no. 14, pp. 1460-1470, 2006.

[8] S. Vardharajula, S. Z. Ali, P. M. Tiwari et al., "Functionalized carbon nanotubes: biomedical applications," International Journal of Nanomedicine, vol. 7, pp. 5361-5374, 2012.

[9] E. Heister, C. Lamprecht, V. Neves et al., "Higher dispersion efficacy of functionalized carbon nanotubes in chemical and biological environments," ACS Nano, vol. 4, no. 5, pp. 26152626, 2010.

[10] C. Richard, F. Balavoine, P. Schultz, T. W. Ebbesen, and C. Mioskowski, "Supramolecular self-assembly of lipid derivatives on carbon nanotubes," Science, vol. 300, no. 5620, pp. $775-778,2003$.

[11] S. Wang, E. S. Humphreys, S.-Y. Chung et al., "Peptides with selective affinity for carbon nanotubes," Nature Materials, vol. 2, no. 3, pp. 196-200, 2003.

[12] M. F. Islam, E. Rojas, D. M. Bergey, A. T. Johnson, and A. G. Yodh, "High weight fraction surfactant solubilization of single-wall carbon nanotubes in water," Nano Letters, vol. 3, no. 2, pp. 269-273, 2003.

[13] P. Petrov, F. Stassin, C. Pagnoulle, and R. Jerome, "Noncovalent functionalization of multi-walled carbon nanotubes by pyrene containing polymers," Chemical Communications, no. 23, pp. 2904-2905, 2003.

[14] M. Zheng, A. Jagota, E. D. Semke et al., "DNA-assisted dispersion and separation of carbon nanotubes," Nature Materials, vol. 2, no. 5, pp. 338-342, 2003.

[15] N. Tagmatarchis and M. Prato, "Functionalization of carbon nanotubes via 1,3-dipolar cycloadditions," Journal of Materials Chemistry, vol. 14, no. 4, pp. 437-439, 2004.

[16] J. L. Hudson, M. J. Casavant, and J. M. Tour, "Water-soluble, exfoliated, nonroping single-wall carbon nanotubes," Journal of the American Chemical Society, vol. 126, no. 36, pp. 11158-11159, 2004.

[17] J. L. Stevens, A. Y. Huang, H. Peng, I. W. Chiang, V. N. Khabashesku, and J. L. Margrave, "Sidewall aminofunctionalization of single-walled carbon nanotubes through fluorination and subsequent reactions with terminal diamines," Nano Letters, vol. 3, no. 3, pp. 331-336, 2003.

[18] A. J. M. Lubag, L. M. De Leon-Rodriguez, S. C. Burgess, and A. D. Sherry, "Noninvasive MRI of-cell function using a $\mathrm{Zn}^{2+}$-responsive contrast agent," Proceedings of the National Academy of Sciences of the United States of America, vol. 108, no. 45, pp. 18400-18405, 2011.

[19] T. Inoue, E. Kozawa, H. Okada et al., "Noninvasive evaluation of kidney hypoxia and fibrosis using magnetic resonance imaging," Journal of the American Society of Nephrology, vol. 22, no. 8, pp. 1429-1434, 2011.

[20] S. Ardhanari, B. Yarlagadda, V. Parikh et al., "Systematic review of non-invasive cardiovascular imaging in the diagnosis of constrictive pericarditis," Indian Heart Journal, vol. 69, no. 1, pp. 57-67, 2017.

[21] D. V. Hingorani, A. S. Bernstein, and M. D. Pagel, "A review of responsive MRI contrast agents: 2005-2014," Contrast Media and Molecular Imaging, vol. 10, no. 4, pp. 245-265, 2015.

[22] J. S. Gustav, J. M. M. Willem, A. F. V. T. Geralda, and N. Klaas, "MRI contrast agents: current status and future perspectives," Anti-Cancer Agents in Medicinal Chemistry, vol. 7, no. 3, pp. 291-305, 2007.

[23] D. Hao, T. Ai, F. Goerner, X. Hu, V. M. Runge, and M. Tweedle, "MRI contrast agents: basic chemistry and safety," Journal of Magnetic Resonance Imaging, vol. 36, no. 5, pp. 1060-1071, 2012.

[24] C. F. G. C. Geraldes and S. Laurent, "Classification and basic properties of contrast agents for magnetic resonance imaging," Contrast Media and Molecular Imaging, vol. 4, no. 1, pp. 1-23, 2009.

[25] M. Rogosnitzky and S. Branch, "Gadolinium-based contrast agent toxicity: a review of known and proposed mechanisms," Biometals, vol. 29, no. 3, pp. 365-376, 2016.

[26] P. Caravan, J. J. Ellison, T. J. McMurry, and R. B. Lauffer, "Gadolinium(III) chelates as MRI contrast agents: structure, dynamics, and applications," Chemical Reviews, vol. 99, no. 9, pp. 2293-2352, 1999.

[27] N. P. Blockley, L. Jiang, A. G. Gardener, C. N. Ludman, S. T. Francis, and P. A. Gowland, "Field strength dependence 
of $\mathrm{R} 1$ and $\mathrm{R} 2 *$ relaxivities of human whole blood to prohance, vasovist, and deoxyhemoglobin," Magnetic Resonance in Medicine, vol. 60, no. 6, pp. 1313-1320, 2008.

[28] M. Rohrer, H. Bauer, J. Mintorovitch, M. Requardt, and H.-J. Weinmann, "Comparison of magnetic properties of MRI contrast media solutions at different magnetic field strengths," Investigative Radiology, vol. 40, no. 11, pp. 715-724, 2005.

[29] B. Sitharaman, K. R. Kissell, K. B. Hartman et al., "Superparamagnetic gadonanotubes are high-performance MRI contrast agents," Chemical Communications, no. 31, pp. 3915-3917, 2005.

[30] J. J. Law, A. Guven, and L. J. Wilson, "Relaxivity enhancement of aquated Tris( $\beta$-diketonate)gadolinium(III) chelates by confinement within ultrashort single-walled carbon nanotubes," Contrast Media and Molecular Imaging, vol. 9, no. 6, pp. 409-412, 2014.

[31] Z. Gu, H. Peng, R. H. Hauge, R. E. Smalley, and J. L. Margrave, "Cutting single-wall carbon nanotubes through fluorination," Nano Letters, vol. 2, no. 9, pp. 1009-1013, 2002.

[32] V. C. Moore, M. S. Strano, E. H. Haroz et al., "Individually suspended single-walled carbon nanotubes in various surfactants," Nano Letters, vol. 3, no. 10, pp. 1379-1382, 2003.

[33] A. Guven, I. A. Rusakova, M. T. Lewis, and L. J. Wilson, "Cisplatin@US-tube carbon nanocapsules for enhanced chemotherapeutic delivery," Biomaterials, vol. 33, no. 5, pp. 1455-1461, 2012.

[34] A. Gizzatov, M. Hernandez-Rivera, V. Keshishian et al., "Surfactant-free $\mathrm{Gd}^{3+}$-ion-containing carbon nanotube MRI contrast agents for stem cell labeling," Nanoscale, vol. 7, no. 28, pp. 12085-12091, 2015.

[35] L. A. Tran, M. Hernández-Rivera, A. N. Berlin et al., “The use of gadolinium-carbon nanostructures to magnetically enhance stem cell retention for cellular cardiomyoplasty," Biomaterials, vol. 35, no. 2, pp. 720-726, 2014.

[36] A. A. Hassan, B. T.-Y. Chan, L. A. Tran et al., "Serinederivatized gadonanotubes as magnetic nanoprobes for intracellular labeling," Contrast Media and Molecular Imaging, vol. 5, pp. 34-38, 2010.

[37] P. Cherukuri, C. J. Gannon, T. K. Leeuw et al., "Mammalian pharmacokinetics of carbon nanotubes using intrinsic nearinfrared fluorescence," Proceedings of the National Academy of Sciences of the United States of America, vol. 103, no. 50, pp. 18882-18886, 2006.

[38] S. Bellucci, M. Chiaretti, P. Onorato et al., "Micro-Raman study of the role of sterilization on carbon nanotubes for biomedical applications," Nanomedicine, vol. 5, no. 2, pp. 209-215, 2010.

[39] R. Sethi, Y. Mackeyev, and L. J. Wilson, "The gadonanotubes revisited: a new frontier in MRI contrast agent design," Inorganica Chimica Acta, vol. 393, pp. 165-172, 2012.

[40] D. E. Newbury and N. W. Ritchie, "Performing elemental microanalysis with high accuracy and high precision by scanning electron microscopy/silicon drift detector energydispersive X-ray spectrometry (SEM/SDD-EDS)," Journal of Materials Science, vol. 50, no. 2, pp. 493-518, 2015. 


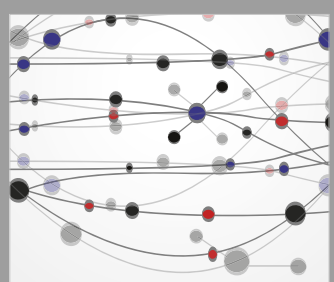

The Scientific World Journal
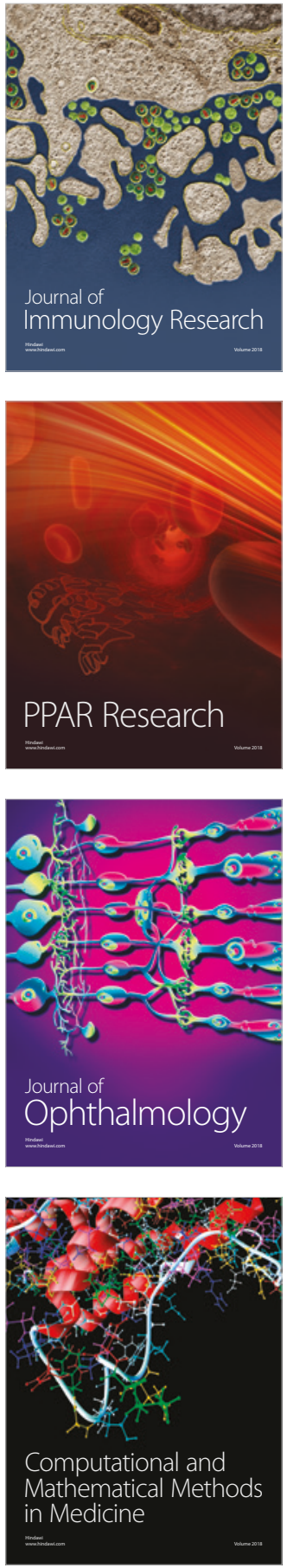

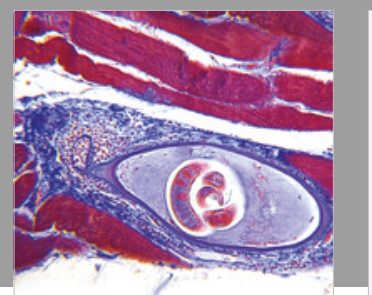

Gastroenterology Research and Practice

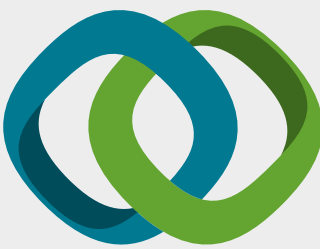

\section{Hindawi}

Submit your manuscripts at

www.hindawi.com
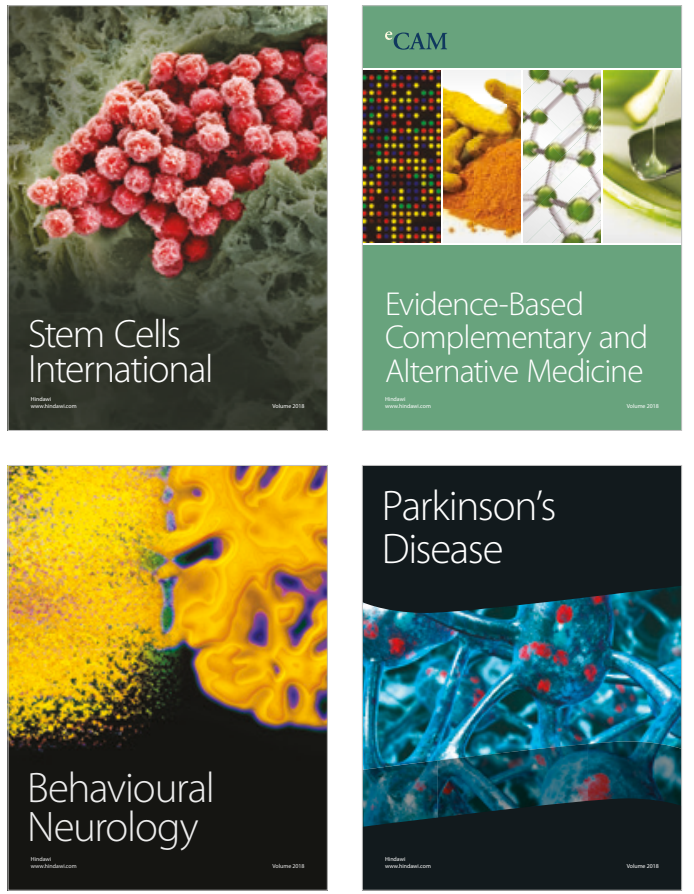

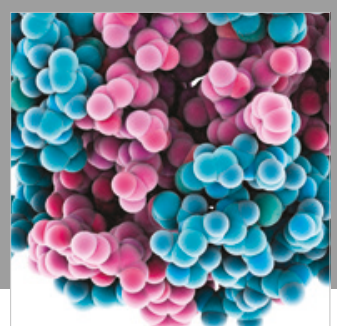

ournal of

Diabetes Research

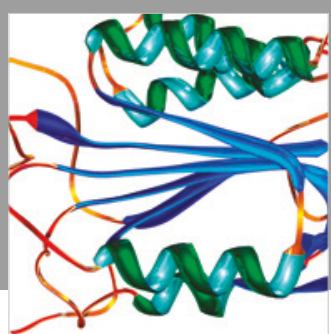

Disease Markers
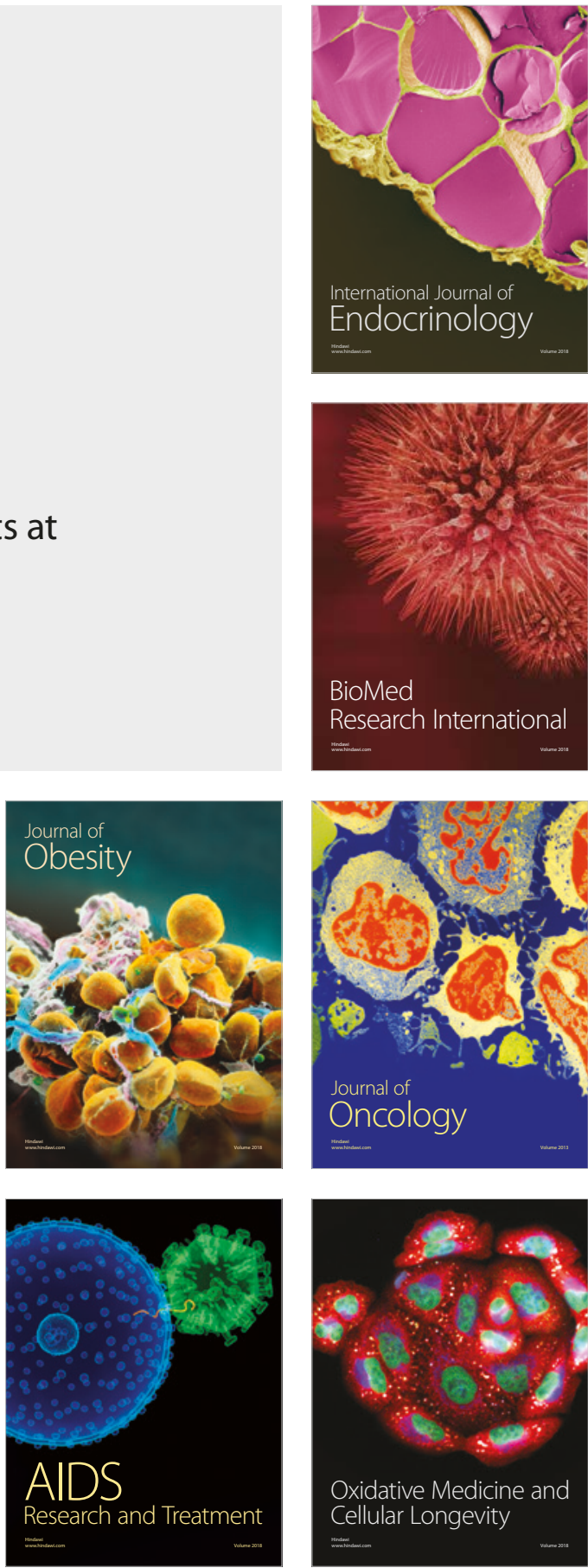